\title{
DARK MATTER AND DARK GAUGE FIELDS
}

\author{
D. V. AHLUWALIA \\ Department of Physics and Astronomy, Rutherford Building \\ University of Canterbury, Private Bag 4800 \\ Christchurch 8020, New Zealand \\ E-mail: dharamvir.ahluwalia-khalilova@canterbury.ac.nz \\ www2.phys.canterbury.ac.nz/editorial/ \\ CHENG-YANG LEE, D. SCHRITT, and T. F. WATSON \\ Department of Physics and Astronomy, Rutherford Building \\ University of Canterbury, Private Bag 4800 \\ Christchurch 8020, New Zealand
}

\begin{abstract}
Following the unexpected theoretical discovery of a mass dimension one fermionic quantum field of spin one half, we now present first results on two local versions. The Dirac and Majorana fields of the standard model of particle physics are supplemented by their natural counterparts in the dark matter sector. The possibility that a mass dimension transmuting symmetry may underlie a new standard model of particle physics is briefly suggested.
\end{abstract}

Keywords: Dirac, Majorana, Elko, Erebus, Nyx, Shakti, Dark Matter

\section{Introduction: Dark matter and its darkness}

The existence of dark matter hints towards new physics, and has often been thought to bring in new symmetries, such as super-symmetry. Yet, such directions can also take us far afield if we have not fully understood the technical and physical content of the well-known continuous and discrete symmetries of the standard model of particle physics. ${ }^{1}$ It is to this possibility that this paper is devoted in the most conservative tradition of our craft.

At present, we do not know the spin of dark matter particles, nor do we have any inkling as to what type of interaction-inducing principle, if any, operates in the dark sector. A highly motivated candidate that answers these questions can suggest experiments that help make properties of dark matter concrete. On the positive side, it is now well established that dark 
matter interacts with the particles of the standard model predominantly via gravity. All other interactions seem to be highly, if not completely, suppressed.

Without calling upon any yet-unobserved symmetries, we invoke the minimal assumption that whatever dark matter is, it must transform in a well-defined manner from one inertial frame to another according to projective irreducible representations of the Poincaré algebra, supplemented by the discrete symmetries of spacetime reflections and charge conjugation. With this conservative ansatz we exploit recently gained insights ${ }^{2,3}$ to construct two fermionic spin one half quantum fields. These carry mass dimension one, and satisfy the canonical locality requirement.

Defining darkness as the property that one set of fields carries limited or no interactions (except gravitational) with another set of fields, these new matter fields are dark with respect to the matter and gauge fields of the standard model (SM) of particle physics; and vice versa.

Identifying the new fields with dark matter (DM), we suggest a massdimension-transmuting symmetry principle that yields the DM sector Lagrangian density without any additional assumptions. This then becomes reminiscent of the super-symmetric paradigm but now the mass-dimensiontransmuting symmetry does not take a fermion to a boson, but a SM fermion of mass dimension three-half to a DM fermion of mass dimension one.

\section{Two new quantum fields}

Here we present all the essential details for the construction of two spin one half quantum fields based upon the dual helicity eigenspinors of the charge conjugation operator $\left(\right.$ Elko ${ }^{\mathrm{a}}$ introduced in references $[2,3]$. The breakthrough that this paper presents, and what constitutes the primary progress since the indicated 2005 publications, is that the quantum fields we now present are local in the canonical sense.

Background - To establish the notation and the working context, we here collect together certain facts and definitions. In case the reader feels a certain element of unfamiliarity, we urge her/him to consult Ref. [3,5] for pedagogic details.

aThe acronym Elko for the eigenspinors of the charge conjugation operator originates from the German Eigenspinoren des Ladungskonjugationsoperators. The reader who wishes to study Elko similarities to and differences from the Majorana spinors may wish to consult references $[3,4]$. 
In the momentum space, dual helicity spinors have the generic form

$$
\lambda(\mathbf{p})=\left(\begin{array}{c}
(\alpha \Theta) \phi_{L}^{*}(\mathbf{p}) \\
\phi_{L}(\mathbf{p})
\end{array}\right)
$$

where $\Theta$ is the Wigner time reversal operator for spin one half and reads

$$
\Theta=\left(\begin{array}{rr}
0 & -1 \\
1 & 0
\end{array}\right)
$$

The $\phi_{L}(\mathbf{p})$ is a massive left-handed (L) Weyl spinor, while $\alpha \Theta \phi_{L}^{*}(p)$ transforms as a right-handed (R) Weyl spinor associated with the same mass. If $\phi_{L}(\mathbf{p})$ carries a given helicity, then $\alpha \Theta \phi_{L}^{*}(p)$ is necessarily endowed with the opposite helicity. The charge conjugation operator $C$ for the $\mathrm{L} \oplus \mathrm{R}$ representation space is

$$
C=\left(\begin{array}{cc}
\mathbb{O} & i \Theta \\
-i \Theta & \mathbb{O}
\end{array}\right) K
$$

where $K$ is the complex conjugation operator (see Ref. [3]). The $\lambda(\mathbf{p})$ become Elko satisfying, $C \lambda(\mathbf{p})= \pm \lambda(\mathbf{p})$, if $\alpha= \pm i$. To construct a complete set of Elko we need the $\mathrm{L} \oplus \mathrm{R}$ boost operator, and a complete dual-helicity basis for Elko at rest; i.e., $\lambda(\mathbf{0})$. The boost operator reads

$$
\kappa=\left(\begin{array}{cc}
\exp \left(\frac{\sigma}{2} \cdot \varphi\right) & \mathbb{O} \\
\mathbb{O} & \exp \left(-\frac{\sigma}{2} \cdot \varphi\right)
\end{array}\right) .
$$

In terms of the energy $E$ and the momentum $\mathbf{p}=p \hat{\mathbf{p}}$ the boost parameter, $\boldsymbol{\varphi}=\varphi \hat{\mathbf{p}}$, is defined as $\cosh (\varphi)=E / m$, and $\sinh (\varphi)=p / m$, where $m$ is the mass of the described particle. Since $(\boldsymbol{\sigma} \cdot \hat{\mathbf{p}})^{2}=\mathbb{I}$, the boost operator is linear in $\mathbf{p}^{\mathrm{b}}$

$$
\kappa=\sqrt{\frac{E+m}{2 m}}\left(\begin{array}{c}
\mathbb{I}+\frac{\boldsymbol{\sigma} \cdot \mathbf{p}}{E+m} \\
\mathbb{O} \\
\mathbb{O}-\frac{\boldsymbol{O} \cdot \mathbf{p}}{E+m}
\end{array}\right) .
$$

Construction of Elko for the two new quantum fields - To construct the required Elko, we must first introduce a basis for the particle description

${ }^{\mathrm{b}}$ It is this fact, when coupled with the observation that the Dirac spinors are eigenspinors of the $\mathrm{L} \oplus \mathrm{R}$ parity operator, that yields the linearity of the Dirac operator $\left(i \gamma^{\mu} \partial_{\mu}-m\right)$. For a complementary discussion, the reader is referred to Sec. 5.5 of reference [6]. 
in its rest frame. Towards this end we shall take $\phi_{L}(\mathbf{0})$ to be eigenspinors of the helicity operator $(\boldsymbol{\sigma} / 2) \cdot \hat{\mathbf{p}}$

$$
\left[\frac{\boldsymbol{\sigma}}{2} \cdot \hat{\mathbf{p}}\right] \phi_{L}^{ \pm}(\mathbf{0})= \pm \frac{1}{2} \phi_{L}^{ \pm}(\mathbf{0}) .
$$

Taking $\hat{\mathbf{p}}=(\sin \theta \cos \phi, \sin \theta \sin \phi, \cos \theta)$, we adopt a certain choice of phase factors so that ${ }^{\mathrm{c}}$

$$
\phi_{L}^{+}(\mathbf{0})=\sqrt{m}\left(\begin{array}{c}
\cos (\theta / 2) e^{-i \phi / 2} \\
\sin (\theta / 2) e^{+i \phi / 2}
\end{array}\right), \phi_{L}^{-}(\mathbf{0})=\sqrt{m}\left(\begin{array}{c}
-\sin (\theta / 2) e^{-i \phi / 2} \\
\cos (\theta / 2) e^{+i \phi / 2}
\end{array}\right) .
$$

The complete basis for Elko that is now required for constructing the indicated quantum fields is

$$
\begin{aligned}
& \xi_{\{-,+\}}(\mathbf{0})=\left.\lambda(\mathbf{0})\right|_{\phi_{L}(\mathbf{0}) \rightarrow \phi_{L}^{+}(\mathbf{0}), \alpha \rightarrow+i} \\
& \xi_{\{+,-\}}(\mathbf{0})=\left.\lambda(\mathbf{0})\right|_{\phi_{L}(\mathbf{0}) \rightarrow \phi_{L}^{-}(\mathbf{0}), \alpha \rightarrow+i} \\
& \zeta_{\{-,+\}}(\mathbf{0})=\left.\lambda(\mathbf{0})\right|_{\phi_{L}(\mathbf{0}) \rightarrow \phi_{L}^{-}(\mathbf{0}), \alpha \rightarrow-i} \\
& \zeta_{\{+,-\}}(\mathbf{0})=-\left.\lambda(\mathbf{0})\right|_{\phi_{L}(\mathbf{0}) \rightarrow \phi_{L}^{+}(\mathbf{0}), \alpha \rightarrow-i}
\end{aligned}
$$

with

$$
\xi_{\{\mp, \pm\}}(\mathbf{p})=\kappa \xi_{\{\mp, \pm\}}(\mathbf{0}), \quad \zeta_{\{\mp, \pm\}}(\mathbf{p})=\kappa \zeta_{\{\mp, \pm\}}(\mathbf{0}) .
$$

The justification for the choice of phases and designations in equations (8-11) is far from trivial; but it is a straightforward generalisation of the reasoning found in Sec. 38 of reference [7] and Sec. 5.5 of reference [6].

If one works with Elko using the Dirac dual, $\bar{\eta}(\mathbf{p})=\eta^{\dagger}(\mathbf{p}) \gamma^{0}$, one finds ${ }^{\mathrm{d}}$ that these carry null norm. ${ }^{9}$ One also encounters problems such as those found in Appendix $\mathrm{P}$ of reference [8]. In fact, the dual-helicity nature of the $\xi(\mathbf{p})$ and $\zeta(\mathbf{p})$ asks for the introduction of a new dual. We shall call it the Elko dual. ${ }^{\mathrm{e}}$ It is defined as

$$
\vec{\eta}_{\{\mp, \pm\}}(\mathbf{p}):=\mp i \eta_{\{ \pm, \mp\}}^{\dagger}(\mathbf{p}) \gamma^{0}
$$

${ }^{\mathrm{c}}$ Please notice that the choice of phases here differs from that given in references $[2,3]$.

${ }^{\mathrm{d}}$ Here $\eta$ stands symbolically for either $\xi$ or $\zeta$.

e The origin of this dual dates back to a preliminary work [10] on the subject. 
where

$$
\gamma^{0}:=\left(\begin{array}{ll}
\mathbb{O} & \mathbb{I} \\
\mathbb{I} & \mathbb{O}
\end{array}\right)
$$

With the Elko dual thus defined, we now have, by construction, the orthonormality relations

$$
\begin{array}{ll}
\bar{\xi}_{\beta}(\mathbf{p}) \xi_{\beta^{\prime}}(\mathbf{p})=+2 m \delta_{\beta \beta^{\prime}}, & \bar{\xi}_{\beta}(\mathbf{p}) \zeta_{\beta^{\prime}}(\mathbf{p})=0 \\
\bar{\zeta}_{\beta}(\mathbf{p}) \zeta_{\beta^{\prime}}(\mathbf{p})=-2 m \delta_{\beta \beta^{\prime}}, & \bar{\zeta}_{\beta}(\mathbf{p}) \xi_{\beta^{\prime}}(\mathbf{p})=0
\end{array}
$$

Here, $\beta$ ranges over two possibilities: $\{+,-\}$ and $\{-,+\}$. The completeness relation is

$$
\frac{1}{2 m} \sum_{\beta}\left[\xi_{\beta}(\mathbf{p}) \bar{\xi}_{\beta}(\mathbf{p})-\zeta_{\beta}(\mathbf{p}) \bar{\zeta}_{\beta}(\mathbf{p})\right]=\mathbb{I} .
$$

The detailed structure underlying the completeness relations resides in the following spin sums

$$
\begin{array}{ll}
\xi_{\beta}(\mathbf{p}) & \stackrel{\xi}{\beta}_{\beta}(\mathbf{p})=+m[\mathbb{I}+\mathcal{G}(\mathbf{p})] \\
\zeta_{\beta}(\mathbf{p}) & \bar{\zeta}_{\beta}(\mathbf{p})=-m[\mathbb{I}-\mathcal{G}(\mathbf{p})]
\end{array}
$$

which together define $\mathcal{G}(\mathbf{p})$. Explicit calculation shows that $\mathcal{G}$ is an odd function of $\mathbf{p}$

$$
\mathcal{G}(\mathbf{p})=-\mathcal{G}(-\mathbf{p})
$$

It must be noted at this stage that the entire set of results is specific to the helicity basis. A careful examination is needed if one wishes to consider a basis in which $\phi_{L}(\mathbf{p})$ are not eigenspinors of the helicity operator but, say, some other operator of the type $(\boldsymbol{\sigma} / 2) \cdot \hat{\mathbf{r}}$; where $\hat{\mathbf{r}}$ is not coincident with $\hat{\mathbf{p}}$. These two bases, as is evident on reflection, represent two physically distinct situations. Our preliminary results confirm that additional work is needed to extract further physical and mathematical content in such situations.

Two new quantum fields based on Elko: Erebus and Nyx - Following Greek mythology about primordial darkness we now introduce two fields which will later be identified with dark matter. We define Erebus as 
$e(x)=\int \frac{d^{3} p}{(2 \pi)^{3}} \frac{1}{\sqrt{2 m E(\mathbf{p})}} \sum_{\beta}\left[c_{\beta}(\mathbf{p}) \xi_{\beta}(\mathbf{p}) e^{-i p_{\mu} x^{\mu}}+d_{\beta}^{\dagger}(\mathbf{p}) \zeta_{\beta}(\mathbf{p}) e^{+i p_{\mu} x^{\mu}}\right]$.

It enjoys the same formal status as the Dirac field constructed from the Dirac spinors. We define Nyx as

$n(x)=\int \frac{d^{3} p}{(2 \pi)^{3}} \frac{1}{\sqrt{2 m E(\mathbf{p})}} \sum_{\beta}\left[c_{\beta}(\mathbf{p}) \xi_{\beta}(\mathbf{p}) e^{-i p_{\mu} x^{\mu}}+c_{\beta}^{\dagger}(\mathbf{p}) \zeta_{\beta}(\mathbf{p}) e^{+i p_{\mu} x^{\mu}}\right]$.

It enjoys at the same formal status as the Majorana field constructed from the Dirac spinors. These formal similarities betray the true physical content of these new fields as we shall discover on deeper analysis. In a nutshell the difference is this: the mass dimension of Erebus and Nyx is one, not three half (see below). It is this aspect that renders them dark with respect to the Dirac and Majorana fields of the standard model of particle physics. ${ }^{f}$ The creation, $c_{\beta}^{\dagger}$ and $d_{\beta}^{\dagger}$, and annihilation, $c_{\beta}$ and $d_{\beta}$, operators that appear in the $e(x)$ and $n(x)$ fields satisfy the standard fermionic anti-commutation relations

$$
\begin{aligned}
& \left\{c_{\beta}(\mathbf{p}), c_{\beta^{\prime}}^{\dagger}\left(\mathbf{p}^{\prime}\right)\right\}=(2 \pi)^{3} \delta_{\beta \beta^{\prime}} \delta^{3}\left(\mathbf{p}-\mathbf{p}^{\prime}\right) \\
& \left\{c_{\beta}(\mathbf{p}), c_{\beta^{\prime}}\left(\mathbf{p}^{\prime}\right)\right\}=\left\{c_{\beta}^{\dagger}(\mathbf{p}), c_{\beta^{\prime}}^{\dagger}\left(\mathbf{p}^{\prime}\right)\right\}=0
\end{aligned}
$$

with similar relations for the $d$ 's.

The Elko duals of Erebus and Nyx fields, $\vec{e}(x)$ and $\vec{n}(x)$, are obtained by the following substitutions in $e(x)$ and $n(x)$

$$
\begin{aligned}
& \xi_{\beta}(\mathbf{p}) \rightarrow \vec{\xi}_{\beta}(\mathbf{p}), \quad \zeta_{\beta}(\mathbf{p}) \rightarrow \vec{\zeta}_{\beta}(\mathbf{p}) \\
& e^{ \pm i p_{\mu} x^{\nu}} \leftrightarrow e^{\mp i p_{\mu} x^{\nu}}, \quad c_{\beta}(\mathbf{p}) \leftrightarrow c_{\beta}^{\dagger}(\mathbf{p}), \quad d_{\beta}(\mathbf{p}) \leftrightarrow d_{\beta}^{\dagger}(\mathbf{p}) .
\end{aligned}
$$

The propagator associated with the Erebus and Nyx fields follows from textbook methods, and is further elaborated in reference [3]. It entails evaluation of \langle|$T\left(e\left(x^{\prime}\right) \vec{e}(x)|\rangle\right.$, and \langle|$T\left(n\left(x^{\prime}\right) \vec{n}(x)|\rangle\right.$, where $T$ is the fermionic time-ordering operator, and |\rangle represents the vacuum state. The result for Erebus as well as Nyx, in terms of spin sums, reads

f The reader may wish to consult reference [3] for the details of this argument. 


$$
\begin{gathered}
S\left(x-x^{\prime}\right)=-\int \frac{d^{3} p}{(2 \pi)^{3}} \quad \frac{i}{2 m E(\mathbf{p})} \sum_{\beta}\left[\theta\left(t^{\prime}-t\right) \xi_{\beta}(\mathbf{p}) \vec{\xi}_{\beta}(\mathbf{p}) e^{-i p_{\mu}\left(x^{\prime \mu}-x^{\mu}\right)}\right. \\
\left.-\theta\left(t-t^{\prime}\right) \zeta_{\beta}(\mathbf{p}) \zeta_{\beta}(\mathbf{p}) e^{+i p_{\mu}\left(x^{\prime \mu}-x^{\mu}\right)}\right]
\end{gathered}
$$

Using the spins sums (18) and (19) yields (again, see reference 3 for additional details)

$$
S\left(x-x^{\prime}\right)=\int \frac{d^{4} p}{(2 \pi)^{4}} e^{i p_{\mu}\left(x^{\mu}-x^{\prime \mu}\right)} \frac{\mathbb{I}+\mathcal{G}(\mathbf{p})}{p_{\mu} p^{\mu}-m^{2}+i \epsilon} .
$$

Here, the limit $\epsilon \rightarrow 0^{+}$is implicit. In view of $(20)$, it is clear that, in the absence of a preferred direction, such as one arising from an external magnetic field, the second term in the above equation identically vanishes. As a result, $S\left(x-x^{\prime}\right)$ reduces to the Klein-Gordon propagator, modulo a $4 \times 4$ multiplicative identity matrix in the $\mathrm{R} \oplus \mathrm{L}$ representation space. Consequently, both the Erebus and Nyx fields carry mass dimension one. This mass dimensionality forbids particles described by these fields to enter $S U(2)_{L}$ doublets of the SM. The fields $e(x)$ and $n(x)$ thus become firstprinciple candidates for dark matter. Following arguments similar to those presented in $[2,3]$ the free Lagrangian densities associated with the Erebus and Nyx fields are

$$
\begin{array}{r}
\mathcal{L}^{\text {Erebus }}=\partial^{\mu} \vec{e}(x) \partial_{\mu} e(x)-m_{e}^{2} \vec{e}(x) e(x) \\
\left.\left.\mathcal{L}^{\mathrm{Nyx}}=\partial^{\mu}\right\urcorner(x) \partial_{\mu} n(x)-m_{n}^{2}\right\urcorner(x) n(x)
\end{array}
$$

Here, we have made it explicit that the Erebus and Nyx fields need not carry the same mass.

\section{A Pause}

A brief pause in the development of our presentation now appears necessary. We physicists are trained to consider Lagrangian density, $\mathcal{L}$, almost as a God-given entity. Granted, one places some restrictions such as that it must be a Lorentz scalar, but beyond these well known caveats that $\mathcal{L}$ rarely is anything but a product of one's genius (such as was the case in Dirac's 1928 paper [11]) or a well-educated guess which often times runs along the lines, "for simplicity let's assume that $\mathcal{L}$ is linear in derivatives, ...". This is all wrong, in essence. Or, so is the lesson that stares us in the face 
of above-derived $\mathcal{L}$ for Erebus and Nyx. In fact, one could have as easily derived $\mathcal{L}^{\text {Dirac }}$ had one started not with the Elko, but with eigenspinors of the parity operator in the $\mathrm{R} \oplus \mathrm{L}$ representation space. ${ }^{\mathrm{g}}$

In a nutshell: The kinematic structure as contained in $\mathcal{L}$ is determined entirely once one agrees that it must be based on a quantum field, and that this quantum field has certain properties under the symmetry operations inherent to the considered representation space. The details are obvious from the presented example of Erebus and Nyx.

\section{Locality structure of the Erebus and Nyx fields}

Having arrived at $\mathcal{L}$ for Erebus and Nyx in (26) and (27) one can immediately obtain the canonically conjugate momenta $\pi(x)$ for these fields. That done, a straightforward, though slightly lengthy calculation involving various Elko 'spin sums' shows that Erebus and Nyx are local. For Erebus we find

$$
\begin{aligned}
& \left\{e(\mathbf{x}, t), \pi\left(\mathbf{x}^{\prime}, t\right)\right\}=i \delta^{3}\left(\mathbf{x}-\mathbf{x}^{\prime}\right) \\
& \left\{e(\mathbf{x}, t), e\left(\mathbf{x}^{\prime}, t\right)\right\}=0,\left\{\pi(\mathbf{x}, t), \pi\left(\mathbf{x}^{\prime}, t\right)\right\}=0
\end{aligned}
$$

An exactly similar set of equal-time anti-commutators exist for Nyx.

These results are in remarkable contrast, and constitute the breakthrough alluded to above, to the non-locality structure presented in references $[2,3]$. These results also provide a counter example to the expectations based on the 1966 work of Lee and Wick. ${ }^{12}$ A resolution of this apparent discrepancy remains to be found.

\section{Towards a standard model with Erebus and Nyx fields}

An intriguing fact that emerges from the above analysis is that the CPT structure of the spin one half $\mathrm{R} \oplus \mathrm{L}$ representation space is far richer than one would have expected. Such an analysis proceeds along similar lines as found in references $[2,3]$ and yields, e.g., $(C P T)^{2}=-\mathbb{I}$ for the Erebus and Nyx fields. The Dirac and Majorana fields are endowed with mass dimension and CPT properties that are in sharp contrast to those of the Erebus and Nyx fields. Therein may lie novel sources of violation of discrete symmetries,

gPlease note that the usual parity operator in the $\mathrm{R} \oplus \mathrm{L}$ representation space can be easily constructed without reference to $\mathcal{L}^{\text {Dirac }}$, or the $\mathcal{L}$ for the Erebus and Nyx fields. If this is not obvious, kindly wait for one of the sequels to this paper. 
such as parity and combined symmetry of charge conjugation and parity, and these may provide insights into such questions as raised by Saunders. ${ }^{13}$

In the present paper we do not further analyse these matters, but instead pose the question: does there exist a fundamental principle that one may invoke to extend the standard model of particle physics to systematically incorporate the Erebus and Nyx fields? We conjecture a symmetry operator $\mathcal{A}$ such that ${ }^{\mathrm{h}}$

$$
\mathcal{A L}^{\text {Dirac }} \mathcal{A}^{-1} \rightarrow \mathcal{L}^{\text {Erebus }}
$$

or, equivalently, $\mathcal{A}^{-1} \mathcal{L}^{\text {Erebus }} \mathcal{A} \rightarrow \mathcal{L}^{\text {Dirac }}{ }^{\text {i }}$ Clearly, such a symmetry, if it exists, transmutes the mass dimension of spin one half fermionic fields from $3 / 2$ to 1 and from 1 to $3 / 2$. The principle of local gauge interactions for the Erebus and Nyx fields in its $U(1)$ form is different from that of the SM: it corresponds to invariance of the Lagrangian density under $|e\rangle \rightarrow$ $\exp \left[i \gamma^{5} \alpha(x)\right]|e\rangle$, and $|n\rangle \rightarrow \exp \left[i \gamma^{5} \alpha(x)\right]|n\rangle$. For the dark gauge fields we choose the name Shakti. This time we have invoked the mythology of the East.

Should $\mathcal{A}$ exist, and be realised by nature, the extended Lagrangian density would form a first-principle extension of the standard model to incorporate dark matter; i.e., if Erebus and Nyx indeed describe dark matter. The latter carries high plausibility given the natural darkness of the $e(x)$ and $n(x)$ fields with respect to the SM fields. We hasten to add that the Erebus and Nyx, just like their SM counterparts, are not, or need not, be self-referentially dark. It is prevented by Shakti.

\section{Concluding remarks}

We have presented two new local quantum fields based on the dual helicity eigenspinors of the charge conjugation operator. These are fermionic, and carry mass dimension one. The latter result provides an ab initio origin of darkness of dark matter, and at the same time suggests the possible existence of a mass dimension transmuting symmetry which may be used to construct an extension of the standard model of particle physics to include the dark matter sector.

The SM matter and gauge fields and their counterparts in Erebus, Nyx, and Shakti may provide two self-referentially luminous sectors of the cosmos

${ }_{\mathrm{h}} \mathrm{A}$ related work has just appeared on the subject. We refer the reader to reference [14] for details.

${ }^{\mathrm{i}}$ With a similar symmetry existing between the Majorana and Nyx fields. 
which, although dark to each other, may be united by Higgs and gravity. Indeed, one may imagine whispering dark rivers and silvery dark moons in the night of the world inhabited by Erebus, Nyx, and Shakti - a world we see not, and yet a world to which owe our existence.

\section{Acknowledgements}

It is our great pleasure to thank all the organisers of "Dark 2007 - Sixth International Heidelberg Conference on Dark Matter in Astro and Particle Physics" for their warm hospitality.

On a personal note we are grateful to two dedicated powerhouses of physics, Hans Klapdor-Kleingrothaus and Irina Krivosheina, who by their relentless pursuit of knowledge and their scholarship have added to physics and its culture the spirit so well captured in Herman Hesse's Das Glasperlenspiel (The Glass Bead Game). This work was inspired by a talk Hans gave more than a decade ago at the Los Alamos National Laboratory and which led the senior author of the present manuscript to investigate Majorana's 1937 paper. ${ }^{15-17}$ That in a series of papers Hans and his collaborators have provided a first glimpse into this beautiful realm attests to the genius of his single-minded pursuits and unique abilities. We give Hans, Irina and their team our very best wishes.

\section{References}

1. E. P. Wigner, Unitary representations of the inhomogeneous Lorentz group including reflections, in Lectures of the Istanbul Summer School of Theoretical Physics (July 16 - August 4, 1962), (Gordon and Breach, New York, 1964).

2. D. V. Ahluwalia-Khalilova and D. Grumiller, Phys. Rev. D 72, 067701 (2005) [arXiv:hep-th/0410192].

3. D. V. Ahluwalia-Khalilova and D. Grumiller, JCAP 0507, 012 (2005) [arXiv:hep-th/0412080].

4. R. da Rocha and W. A. J. Rodrigues, Mod. Phys. Lett. A 21, 65 (2006) [arXiv:math-ph/0506075].

5. L. H. Ryder, Quantum Field Theory, (Cambridge University Press, Cambridge, 1996).

6. S. Weinberg, The quantum theory of fields. Vol. 1: Foundations, (Cambridge University Press, Cambridge, 1995).

7. M. Srednicki, Quantum field theory, (Cambridge University Press, Cambridge, 2007).

8. I. J. R. Aitchison and A. J. G. Hey, Gauge theories in particle physics: A practical introduction. Vol. 2: (IOP Publishers, Bristol, 2004).

9. D. V. Ahluwalia, Int. J. Mod. Phys. A 11, 1855 (1996) [arXiv:hepth/9409134]. 
10. D. V. Ahluwalia-Khalilova, Extended set of Majorana spinors, a new dispersion relation, and a preferred frame, arXiv:hep-ph/0305336. It is to be noted that many of the results presented in this preprint were later collaboratively developed with D. Grumiller, and published in reference [2,3].

11. P. A. M. Dirac, Proc. Roy. Soc. Lond. A 117, 610 (1928).

12. T. D. Lee and G. C. Wick, Phys. Rev. 148, 1385 (1966).

13. S. Saunders, "Mirroring as an A Priori Symmetry," Philosophy of Science (in press).

14. R. da Rocha and J. M. Hoff da Silva, J. Math. Phys. 48, 123517 (2007) [arXiv:0711.1103 [math-ph]].

15. E. Majorana, Nuovo Cim. 14, 171 (1937).

16. H. V. Klapdor-Kleingrothaus, A. Dietz, H. L. Harney and I. V. Krivosheina, Mod. Phys. Lett. A 16, 2409 (2001) [arXiv:hep-ph/0201231].

17. H. V. Klapdor-Kleingrothaus and I. V. Krivosheina, Mod. Phys. Lett. A 21, 1547 (2006). 